PROCEEDINGS OF THE

AMERICAN MATHEMATICAL SOCIETY

Volume 129, Number 11, Pages 3285-3295

S 0002-9939(01)06057-9

Article electronically published on April 9, 2001

\title{
SPECTRAL RADIUS OF THE SAMPLING OPERATOR WITH CONTINUOUS SYMBOL
}

\author{
MARK C. HO
}

(Communicated by David R. Larson)

\begin{abstract}
Let $\varphi(\theta) \sim \sum_{-\infty}^{\infty} a_{k} e^{i k \theta}$ (where $a_{k}$ is the $k$-th Fourier coefficient of $\varphi$ ) be a bounded measurable function on the unit circle $\mathbf{T}$. Consider the operator $S_{\varphi}(m, n)$ on $L^{2}(\mathbf{T})$ whose matrix with respect to the standard basis $\left\{e^{i k \theta}: k \in \mathbf{Z}\right\}$ is given by $\left(a_{m i-n j}\right)_{i, j \in \mathbf{Z}}$. In this paper, we give upper and lower bound estimation for $r\left(S_{\varphi}(m, n)\right)$, the spectral radius of $S_{\varphi}(m, n)$. Furthermore, we will show that in some cases (for example, if $\varphi$ is continuous on $\mathbf{T}$ and $\varphi>0)$, the spectral radius of $S_{\varphi}(m, n)$ can be computed exactly in terms of roots of the norms of some finite Toeplitz matrices.
\end{abstract}

\section{INTRODUCTION}

Let us consider the Hilbert space $L^{2}(\mathbf{T})=\left\{f: \int_{\mathbf{T}}|f|^{2}<\infty\right\}$ with the usual inner product $\langle f, g\rangle=\int_{\mathbf{T}} f \bar{g}$ and a bounded measurable function $\varphi(\theta) \sim \sum_{-\infty}^{\infty} a_{k} e^{i k \theta}$ (where $a_{k}$ is the $k$-th Fourier coefficient of $\varphi$ ) on the unit circle $\mathbf{T}=\left\{e^{i \theta}: \theta \in \mathbf{R}\right\}$. We will denote the operator of multiplication by $\varphi$ as $M_{\varphi}$. It is well-known that the matrix of $M_{\varphi}$ with respect to the standard basis $\left\{e^{i k \theta}: k \in \mathbf{Z}\right\}$ is given by $\left(a_{i-j}\right)_{i, j \in \mathbf{Z}}$. It is also known that the norm and spectral radius of $M_{\varphi}$ are the same, given by $\|\varphi\|_{\infty}=\sup _{0 \leq \theta<2 \pi}\left|\varphi\left(e^{i \theta}\right)\right|$.

Let $m, n \in \mathbf{N}$. The sampling operator $S_{\varphi}(m, n)$ with symbol $\varphi$ and parameter $m, n$ is the operator on $L^{2}(\mathbf{T})$ whose matrix representation with respect to $\left\{e^{i k \theta}: k \in \mathbf{Z}\right\}$ is obtained by "keeping" every $m$-th row and $n$-th column of the doubly infinite matrix of $M_{\varphi}$, i.e., the matrix $\left(a_{m i-n j}\right)_{i, j \in \mathbf{Z}}$.

A sampling operator arises from considering some alternative for a given interpolation scheme in approximation theory. Let $f$ be a function on $\mathbf{R}$ whose values (or sometimes called the outputs of $f$ ) are known at equally spaced points, say for example, the integers, and we are given an interpolation scheme that allows us to estimate the values of $f$ at, say, the set $\left\{\frac{k}{m}: k \in \mathbf{Z}\right\}$ while keeping the same values of $f$ at the integers. The alternative scheme here is for one to only approximate the values of $f$ at the subset $\left\{\frac{n j}{m}: j \in \mathbf{Z}\right\}$ (for the details on this subject one can check 8]). To approach this problem from the operator theory point of view, one can formulate the problem as follows: Let $\left\{y_{0}(k)\right\}_{-\infty}^{\infty}$ be the values of $f$ at the integers and let $\left\{y_{1}(j)\right\}_{-\infty}^{\infty}$ be the estimates for $f$ on $\left\{\frac{n j}{m}: j \in \mathbf{Z}\right\}$, with $y_{0}(0)=y_{1}(0)=f(0)$.

Received by the editors December 8, 1999 and, in revised form, March 12, 2000.

1991 Mathematics Subject Classification. Primary 42C15, 47C35, 47C38. 
Then

$$
y_{1}(j)=\sum_{k=-\infty}^{\infty} a_{m j-n k} y_{0}(k)
$$

where $\varphi(\theta) \sim \sum_{-\infty}^{\infty} a_{k} e^{i k \theta}$ is usually a real-valued function on $\mathbf{T}$ associated with the particular scheme chosen.

The spectral radius of the sampling operator $S_{\varphi}(m, n)$ is known to have close ties to the so-called "smoothness index" of the interpolation scheme that $\varphi$ is associated with. For example, in the case of the dyadic wavelets in $L^{2}(\mathbf{R})$ (which corresponds to the interpolation scheme for half integers $\left\{\frac{k}{2}: k \in \mathbf{Z}\right\}$ ), usually one first obtains a smooth solution $f$ in $L^{2}(\mathbf{T})$ of the equation

$$
f(t)=\sum_{k} c_{k} f(2 t-k),
$$

where $c_{k}$ is the $k$-th Fourier coefficient of a trigonometric polynomial $\varphi$ on $\mathbf{T}$, and then constructs the so-called mother function from $f$ to generate the wavelet basis with desired properties. In order to determine the regularity of the generated wavelet basis, and consequently the smoothness of $f$, one calculates the quantity

$$
s_{0}=M-\log _{2} r\left(S_{\tilde{\varphi}}(2,1)\right)-\frac{1}{2},
$$

where $s_{0}$ is the so-called Sobolev exponent of $f$ and $M$ is the multiplicity of $\varphi$ at $\theta=\pi$. The term $r\left(S_{\tilde{\varphi}}(2,1)\right)$ is precisely the spectral radius for $S_{\tilde{\varphi}}(2,1)$ on $L^{2}(\mathbf{T})$ and $\tilde{\varphi}$ is obtained basically by factoring out the zeros of $\varphi$ at $\theta=\pi$ up to its multiplicity. For information on the subjects concerning the estimation for the regularity of wavelet basis see, for example, 1, [2], 4], 5] and [9].

The purpose of this article is to give an explicit formula to compute $r\left(S_{\varphi}(m, n)\right)$ in terms of the asymptotic behavior of the norms of some finite Toeplitz matrices if $\varphi$ is a positive continuous function on $\mathbf{T}$ (Theorem 3.3).

\section{NORMS FOR THE SAMPLING OPERATORS}

Throughout this article we will use the notation $\varphi(\theta)=\varphi\left(e^{i \theta}\right), \theta \in \mathbf{R}$. Let $\tau_{m}$ be the continuous mapping defined on $\mathbf{T}$ by $\tau_{m}: e^{i \theta} \rightarrow e^{i m \theta}$. Consider the composition operator $C_{m}=C_{\tau_{m}}$ on $L^{2}(\mathbf{T})$ (i.e., $C_{m} f=f \circ \tau_{m}$ ) and the average operator $R_{m}$ defined by

$$
\left(R_{m} f\right)(\theta):=\frac{1}{m} \sum_{l=0}^{m-1} f\left(\frac{\theta+2 l \pi}{m}\right) \quad \text { a.e. } \theta \in \mathbf{R}
$$

for all $f \in L^{2}(\mathbf{T})$. It is easy to see that $C_{m}$ is an isometry and $R_{m}$ is a partial isometry. Moreover, $R_{m}=C_{m}^{*}$ for every $m$. It also follows by the definitions that

$$
R_{m} e^{i k \theta}= \begin{cases}e^{i s \theta} & \text { if } k=m s \\ 0 & \text { otherwise. }\end{cases}
$$

It is also easy to check, with this notation, that we have

$$
S_{\varphi}(m, n)=R_{m} M_{\varphi} C_{n} .
$$

From this, it is evident that $S_{\varphi}(m, n)$ is a bounded operator on $L^{2}(\mathbf{T})$ since $\left\|S_{\varphi}(m, n)\right\|=\left\|R_{m} M_{\varphi} C_{n}\right\| \leq\left\|M_{\varphi}\right\| \leq\|\varphi\|_{\infty}$. 
The following are some useful facts about $R_{m}$ and $C_{n}(m, n \in \mathbf{N})$ :

(2,1) $R_{m} C_{m}=I$ and $C_{m} R_{m}=P_{m}$, where $P_{m}$ is the projection from $L^{2}(\mathbf{T})$ onto the closed span of $\left\{e^{i m k \theta}: k \in \mathbf{Z}\right\}$. Also, it is easy to see that $R_{m} R_{n}=R_{m n}$ and $C_{m} C_{n}=C_{m n}$.

(2, 2) $P_{m}=\frac{1}{m} \sum_{l=0}^{m-1} \mathcal{L}_{\zeta_{m}^{l}}$, where $\mathcal{L}_{\zeta}$ is the rotation operator $\left(\mathcal{L}_{\zeta} f\right)(\theta)=f\left(\zeta e^{i \theta}\right)$ $(\zeta \in \mathbf{C})$ and $\zeta_{m}=e^{i \frac{2 \pi}{m}}$.

(2,3) For any $\varphi$ in $L^{\infty}(\mathbf{T}), R_{m} M_{\varphi} C_{m}=M_{\psi}$, where $\psi=R_{m} \varphi$.

$(2,4)$ From the computation

$$
\begin{aligned}
\left\|M_{\bar{\varphi}} C_{m} f\right\|_{2}^{2} & =\left\langle M_{\bar{\varphi}} C_{m} f, M_{\bar{\varphi}} C_{m} f\right\rangle \\
& =\left\langle R_{m} M_{|\varphi|^{2}} C_{m} f, f\right\rangle \\
& =\left\langle M_{\psi} f, f\right\rangle,
\end{aligned}
$$

where $\psi=R_{m}\left(|\varphi|^{2}\right)$, one has $\left\|R_{m} M_{\varphi}\right\|^{2}=\left\|M_{\bar{\varphi}} C_{m}\right\|^{2}=\left\|R_{m}\left(|\varphi|^{2}\right)\right\|_{\infty}$.

(2.5) $M_{\varphi} R_{m}=R_{m} M_{\varphi \circ \tau_{m}}$ and $C_{m} M_{\varphi}=M_{\varphi \circ \tau_{m}} C_{m}$ for any $m \in \mathbf{N}$ and any $\varphi \in L^{\infty}(\mathbf{T})$ (in fact, we have $C_{\tau} M_{\varphi}=M_{\varphi \circ \tau} C_{\tau}$ in general, for any $\tau$ on a compact metric space $X$ that induces a bounded $C_{\tau}$ on $L^{2}(X, d \mu)$ with respect to the measure $\mu$ ).

Now we compute the norm for $S_{\varphi}(m, n)^{k}, k \in \mathbf{N}$ :

Lemma 2.1. Given $m, n$ in $\mathbf{N}$ such that $(m, n)=1($ where $(m, n)=$ g.c.d. $(m, n))$. Then $C_{m}$ and $R_{n}$ commute, i.e., $C_{m} R_{n}=R_{n} C_{m}$.

Proof. Simply check the equality $C_{m} R_{n} e^{i k \theta}=R_{n} C_{m} e^{i k \theta}$ for all $k \in \mathbf{Z}$, using the fact that $(m, n)=1$.

Lemma 2.2. If $(m, n)=1$, then for $f \in L^{2}(\mathbf{T})$ we have

$$
\left\|S_{\varphi}(m, n) f\right\|^{2}=\sum_{k=0}^{n-1}\left\|R_{m} M_{\varphi_{k}} f\right\|_{2}^{2}
$$

where

$$
\varphi_{k}(\theta)=e^{-i k a \theta} R_{n}\left(e^{i k \theta} \varphi\right)=e^{-i k a \theta} \sum_{j=0}^{n-1} e^{i k\left(\frac{\theta+2 j \pi}{n}\right)} \varphi\left(\frac{\theta+2 j \pi}{n}\right)
$$

for some a such that na $\equiv 1 \bmod m$. Here $\|f\|_{2}=\left(\int_{\mathbf{T}}|f|^{2}\right)^{\frac{1}{2}}$.

Proof. First let us adopt a decomposition of $M_{\varphi}$ due to Zizler in Theorem 8, [10]. For each $0 \leq k \leq n-1$, let $H_{k}=\bigvee\left\{e^{i(k+n a) \theta}: a \in \mathbf{Z}\right\}$ and consider $h_{k}=$ $e^{-i k \theta} C_{n} R_{n}\left(e^{i k \theta} \varphi\right)$. Then it follows from (1) that $\varphi=h_{0}+h_{1} \cdots+h_{n-1}$ and $M_{h_{k}} C_{n} f \in H_{k}$ for all $k$. Therefore, $M_{\varphi} C_{n}=M_{h_{0}} C_{n}+M_{h_{1}} C_{n}+\cdots+M_{h_{n-1}} C_{n}$ and $\left\langle M_{h_{s}} C_{n} f, M_{h_{t}} C_{n} f\right\rangle=0$ for all $f \in L^{2}(\mathbf{T})$ if $s \neq t(0 \leq s, t \leq n-1)$.

On the other hand, it also follows from (1) that $R_{m}\left(e^{i(s+n a) \theta}\right) \neq 0$ only if $s+n a=m(t+n b)$ for some $0 \leq t \leq n-1$ and $b \in \mathbf{Z}$ and consequently, only if $s \equiv m t \bmod n$ for some $0 \leq t \leq n-1$. Suppose that $0 \leq s_{1} \neq s_{2} \leq n-1$ and $s_{1} \equiv m t_{1} \bmod n, s_{2} \equiv m t_{2} \bmod n$ for some $0 \leq t_{1}, t_{2} \leq n-1$. Then $t_{1} \neq t_{2}$. This means that $\left\langle R_{m} M_{h_{s}} C_{n} f, R_{m} M_{h_{t}} C_{n} f\right\rangle=0$ for all $f \in \overline{L^{2}}(\mathbf{T})$ if $s \neq t$, and from this 
we get

$$
\left\|S_{\varphi}(m, n) f\right\|_{2}^{2}=\sum_{k=0}^{n-1}\left\|R_{m} M_{h_{k}} C_{n} f\right\|_{2}^{2}, f \in L^{2}(\mathbf{T}) .
$$

Now let $\alpha_{k} \in \mathbf{Z}$ such that $n \alpha_{k}+k \equiv 0 \bmod m$ for each $k$. Then

$$
\left\|R_{m}\left(e^{i\left(k+n \alpha_{k}\right) \theta} g\right)\right\|_{2}=\left\|R_{m} g\right\|_{2}
$$

for any $g \in H_{k}$ and for each $k$. Therefore, for any $f \in L^{2}(\mathbf{T})$,

$$
\begin{aligned}
\left\|R_{m}\left(e^{i\left(k+n \alpha_{k}\right) \theta} M_{h_{k}} C_{n} f\right)\right\|_{2} & =\left\|R_{m} C_{n}\left(e^{\alpha_{k} \theta} R_{n}\left(e^{i k \theta} \varphi\right) f\right)\right\|_{2} \\
& =\left\|C_{n} R_{m}\left(e^{\alpha_{k} \theta} R_{n}\left(e^{i k \theta} \varphi\right) f\right)\right\|_{2} \\
& =\left\|R_{m}\left(e^{\alpha_{k} \theta} R_{n}\left(e^{i k \theta} \varphi\right) f\right)\right\|_{2}
\end{aligned}
$$

for each $k$. Notice that the above equations follow from the definitions, Lemma 2.1 and the fact that $C_{n}$ is an isometry.

To finish the proof, notice that since $(m, n)=1$, there are $a, b \in \mathbf{Z}$ so that $n a+m b=1$. This means that $n a \equiv 1 \bmod m$, and therefore $n(-k a)+k \equiv 0$ $\bmod m$ for each $k$, which implies that we may choose $\alpha_{k}=-k a$.

Remark. Here we would like to point out that $R_{n}\left(|\varphi|^{2}\right)=\sum_{k=0}^{n-1} R_{n}\left(\left|h_{k}\right|^{2}\right)=$ $\sum_{k=0}^{n-1}\left|\varphi_{k}\right|^{2}$. The following is a simple proof: Write

$$
\varphi(\theta)=\sum_{k=0}^{n-1} h_{k}(\theta)=\sum_{k=0}^{n-1} e^{-i k \theta} \varphi_{k}(\theta)
$$

then

$$
\begin{aligned}
R_{n}\left(|\varphi|^{2}\right)(\theta) & =\sum_{k=0}^{n-1}\left|\varphi\left(\frac{\theta+2 k \pi}{n}\right)\right|^{2} \\
& =\frac{1}{n} \sum_{s, s^{\prime}=0}^{n-1} \varphi_{s}(\theta) \bar{\varphi}_{s^{\prime}}(\theta) e^{\frac{-i\left(s-s^{\prime}\right) \theta}{n}} \sum_{k=0}^{n-1} e^{\frac{-i 2\left(s-s^{\prime}\right) k \pi}{n}} \\
& =\sum_{k=0}^{n-1}|\varphi(\theta)|^{2}=\sum_{k=0}^{n-1}\left|h_{k}(\theta)\right|^{2} .
\end{aligned}
$$

Theorem 2.3. Suppose that $(m, n)=r$. Then $\left\|S_{\varphi}(m, n)\right\|^{2}$ equals the essential supreme of

$$
\|S(\theta)\|
$$

over $[0,2 \pi)$, where $S(\theta)$ is a $q \times q$ positive definite matrix on $\mathbf{C}^{p}$ whose st-th entry is the multiplication by $R_{p}\left(\psi_{s} \bar{\psi}_{t}\right)(\theta)$ a.e. $\theta \in[0,2 \pi)(0 \leq s, t \leq q-1)$, with $m=p r$, $n=q r, \psi=R_{r} \varphi$ and $\psi_{s}$ is defined by

$$
\psi_{s}(\theta)=e^{-i s a \theta} R_{q}\left(e^{i s \theta} \psi\right)=e^{-i s a \theta} \sum_{j=0}^{q-1} e^{i s\left(\frac{\theta+2 j \pi}{q}\right)} \psi\left(\frac{\theta+2 j \pi}{q}\right)
$$

for some a so that $q a \equiv 1 \bmod p$. 
Proof. First observe $S_{\varphi}(m, n)=R_{m} M_{\varphi} C_{n}=R_{p}\left(R_{r} M_{\varphi} C_{r}\right) C_{q}=R_{p} M_{\psi} C_{q}$ (see (2.1)-(2.5)). Therefore, by Lemma 2.2 we have

$$
\left\|S_{\varphi}(m, n)\right\|^{2}=\sup _{\|f\|_{2}=1} \sum_{k=0}^{q-1}\left\|R_{p} M_{\psi_{k}} f\right\|_{2}^{2} .
$$

Now consider the operator $T$ on $K=\bigoplus_{1}^{q} L^{2}(\mathbf{T})$ defined by

$$
T:=\left(\begin{array}{ccccc}
R_{p} M_{\psi_{0}} & \mathbf{0} & \cdots & \cdots & \mathbf{0} \\
\vdots & \vdots & \ddots & & \vdots \\
\vdots & \vdots & & \ddots & \vdots \\
R_{p} M_{\psi_{q-1}} & \mathbf{0} & \cdots & \cdots & \mathbf{0}
\end{array}\right) .
$$

It is easy to see that $\|T\|^{2}=\left\|S_{\varphi}(m, n)\right\|^{2}$. Therefore, the proof follows from (2]3), $\|T\|^{2}=\left\|T^{*}\right\|^{2}=\left\|T T^{*}\right\|$ and the fact that $T T^{*}$ is a block multiplication operator on $K$.

Remark. There are two immediate consequences of Theorem 2.3. First, we are now able to obtain upper bound for $\left\|S_{\varphi}(m, n)\right\|$. Specifically, since for a.e. $\theta, S(\theta)$ is positive definite, we have (see the remark after Lemma 2.2)

$$
\|S(\theta)\| \leq \operatorname{Tr}(S(\theta))=R_{p q}\left(|\psi|^{2}\right)(\theta) \text { a.e. } \theta
$$

(where $\operatorname{Tr}(A)$ is the trace of $A$ ). Therefore, $\left\|S_{\varphi}(m, n)\right\| \leq\left\|R_{p q}\left(|\psi|^{2}\right)\right\|_{\infty}^{\frac{1}{2}}$. Second, since the largest eigenvalue $\lambda(\theta)$ of $S(\theta)$ satisfies

$$
\lambda(\theta) \geq q^{-1} \operatorname{Tr}(S(\theta))=q^{-1} R_{p q}\left(|\psi|^{2}\right)(\theta) \text { a.e. } \theta,
$$

$\left\|S_{\varphi}(m, n)\right\|$ is bounded below by $q^{-\frac{1}{2}}\left\|R_{p q}\left(|\psi|^{2}\right)\right\|_{\infty}^{\frac{1}{2}}$. The readers can find detailed discussion on the subject of positive definite matrices in, for example, Chapter 7 in 6 . Here we point out that the upper bound above for $\left\|S_{\varphi}(m, n)\right\|$ was first obtained by Zizler in [10].

Combining $S_{\varphi}(m, n)^{k}=R_{p^{k}} M_{\psi_{k}} C_{q^{k}}$ (by (2.4)), (2.5), Lemma2.1 and Theorem 2.3, we immediately obtain

Proposition 2.4. Suppose that $(m, n)=r$. Then $\left\|S_{\varphi}(m, n)^{k}\right\|^{2}$ equals the essential supreme of

$$
\left\|S_{k}(\theta)\right\|
$$

over $[0,2 \pi)$, where $S_{k}(\theta)$ is a $q^{k} \times q^{k}$ positive definite matrix on $\mathbf{C}^{p^{k}}$ whose entries are given by $R_{p^{k}}\left(\psi_{k, s} \bar{\psi}_{k, t}\right)(\theta)$ a.e. $\theta \in[0,2 \pi)\left(0 \leq s, t \leq p^{k}-1\right)$, with $m=p r$, $n=q r, \psi=R_{r} \varphi, \psi_{k, s}=e^{-i s a_{k} \theta} R_{p^{k}}\left(e^{i s \theta} \psi_{k}\right)$ for all $s$, where

$$
\psi_{k}(\theta)=\prod_{\gamma=0}^{k-1} \psi\left(p^{\gamma} q^{k-1-\gamma} \theta\right)
$$

and $q^{k} a_{k} \equiv 1 \bmod p^{k}$.

Corollary 2.5. $r\left(S_{\varphi}(m, n)\right)>0$ if $\log |\psi| \in L^{1}(\mathbf{T})$. 
Proof. By Proposition 2.4 and the remark after Theorem 2.3, we have

$$
\begin{aligned}
\left\|S_{\varphi}(m, n)^{k}\right\|^{2} & \geq \frac{1}{q^{k}}\left\|R_{p^{k} q^{k}}\left(\left|\psi_{k}\right|^{2}\right)\right\|_{\infty} \geq \frac{1}{q^{k}} \int_{0}^{2 \pi} R_{p^{k} q^{k}}\left(\left|\psi_{k}\right|^{2}\right) \frac{d \theta}{2 \pi} \\
& =\frac{1}{q^{k}} \int_{0}^{2 \pi}\left|\psi_{k}\right|^{2} \frac{d \theta}{2 \pi} \geq \frac{1}{q^{k}} \exp \left(\int_{0}^{2 \pi} \log \left|\psi_{k}\right|^{2} \frac{d \theta}{2 \pi}\right) \\
& =\frac{1}{q^{k}} \exp \left(k \int_{0}^{2 \pi} \log |\psi|^{2} \frac{d \theta}{2 \pi}\right) .
\end{aligned}
$$

This means that

$$
r\left(S_{\varphi}(m, n)\right)=\lim _{k \rightarrow \infty}\left\|S_{\varphi}(m, n)^{k}\right\|^{\frac{1}{k}} \geq \frac{1}{\sqrt{q}} \exp \left(\int_{0}^{2 \pi} \log |\psi| \frac{d \theta}{2 \pi}\right),
$$

and therefore the result follows from the assumption.

\section{Spectral Radius computation for $S_{\varphi}(m, n)$}

As a consequence of Proposition 2.4 we see that for any $\varphi \in L^{\infty}(\mathbf{T})$

$$
\frac{1}{\sqrt{q}} \limsup _{k \rightarrow \infty}\left\|R_{p^{k} q^{k}}\left(\left|\psi_{k}\right|^{2}\right)\right\|_{\infty}^{\frac{1}{2 k}} \leq r\left(S_{\varphi}(m, n)\right) \leq \liminf _{k \rightarrow \infty}\left\|R_{p^{k} q^{k}}\left(\left|\psi_{k}\right|^{2}\right)\right\|_{\infty}^{\frac{1}{2 k}}
$$

(it can be shown that $\lim _{k \rightarrow \infty}\left\|R_{p^{k} q^{k}}\left(\left|\psi_{k}\right|^{2}\right)\right\|_{\infty}^{\frac{1}{2 k}}$ actually exists). But there is an obvious problem for these upper and lower bounds estimates, that is, they may be far apart when $q$ is large. However, for the case of estimating $r\left(S_{\varphi}(m, n)\right)$ when $\varphi$ is continuous and positive, we will show that we can do much better. Before we begin, let us first introduce some very useful notation:

Definition. Let $\left\{f_{k}\right\}$ and $\left\{g_{k}\right\}$ be two sequences of positive functions on $\mathbf{T}$. We say that $f_{k}$ is comparable to $g_{k}$, denoted by $f_{k} \approx g_{k}$, if given any $0<\delta<1<\rho$, there exists constant $c>0$ such that

$$
c^{-1} \delta^{k} f_{k} \leq g_{k} \leq c \rho^{k} f_{k} \quad \text { uniformly }
$$

if $k \geq 1$. Two sequences of positive numbers $\left\{\alpha_{k}\left(s_{k}(1), \cdots, s_{k}(n)\right): a_{k} \leq s_{k}(i) \leq\right.$ $\left.b_{k}, a_{k}, b_{k} \in \mathbf{N}\right\}$ and $\left\{\beta_{k}\left(s_{k}(1), \cdots, s_{k}(n)\right): a_{k} \leq s_{k}(i) \leq b_{k}, a_{k}, b_{k} \in \mathbf{N}\right\}$ with multiple indices are said to be comparable uniformly in $k$ (with the same notation) if they satisfy (2) for some constants that, in addition, do not depend on $1 \leq i \leq n$. Two sequences of positive numbers are said to be comparable if they satisfy (2) for some constants when they are regarded as either sequences of constant functions or a special case for sequences with multiple indices.

It is easy to see that the relation " $\approx$ " is transitive, i.e., if $f_{k} \approx g_{k}$ and $g_{k} \approx h_{k}$, then $f_{k} \approx h_{k}$. Also, if $f_{k} \approx g_{k}$, then $\frac{\sqrt[k]{f_{k}}}{\sqrt[k]{g_{k}}} \rightarrow 1$ uniformly on $\mathbf{T}$ as $k \rightarrow \infty$ since $\varepsilon$ and $\rho$ are arbitrary. A similar result also holds for sequences.

Lemma 3.1. Suppose that $\psi \in C(\mathbf{T})$ and $|\psi|>0$. If $p>q$, then

$$
\left|\psi_{k}(\theta+\alpha)\right| \approx\left|\psi_{k}(\theta)\right|
$$

uniformly over $\mathbf{R}$ if $|\alpha| \leq \frac{2 \pi}{p^{k}}$, where $\psi_{k}(\theta)=\prod_{\gamma=0}^{k-1} \psi\left(p^{\gamma} q^{k-1-\gamma} \theta\right)$.

Proof. Since $\mathbf{T}$ is compact and $|\psi|>0,|\psi|$ is uniformly continuous in the sense that given any $0<\delta<1<\rho$, there exists $\varepsilon>0$ so that $\delta\left|\psi\left(\theta_{1}\right)\right|<\left|\psi\left(\theta_{2}\right)\right|<\rho\left|\psi\left(\theta_{1}\right)\right|$ if $\left|\theta_{1}-\theta_{2}\right|<\varepsilon$. On the other hand, choose $k_{0}$ large enough so that $2 \pi q^{k-s}<\varepsilon p^{k-s}$ 
whenever $k-s \geq k_{0}-s_{0}$ for some $s_{0}$ depending only on $p$ and $q$. This is possible since $p>q$. It follows that for $\theta \in \mathbf{R}$,

$$
K^{-s_{0}} \delta^{k-s_{0}}\left|\psi_{k}(\theta+\alpha)\right| \leq\left|\psi_{k}(\theta)\right| \leq K^{s_{0}} \rho^{k-s_{0}}\left|\psi_{k}(\theta+\alpha)\right|
$$

if $k \geq k_{0}$, where $K=\frac{m}{M}$ and $M$ and $m$ are the maximum and minimum of $|\psi|$ on $\mathbf{T}$, respectively. This completes the proof.

Lemma 3.2. Let $p>q$ in $\mathbf{N},(p, q)=1$ and $\psi>0$ in $C(\mathbf{T})$. For each $k$, let $\Psi_{k}$ be the step function on $\mathbf{T}$ satisfying

$$
\Psi_{k}(\theta)=\psi_{k}\left(\frac{2 \pi l}{p^{k}}\right), \quad 0 \leq l \leq p^{k}-1,
$$

if $\theta \equiv \phi \bmod 2 \pi$ for some $\frac{2 \pi b_{l}}{p^{k}} \leq \phi<\frac{2 \pi\left(b_{l}+1\right)}{p^{k}}$, where $0 \leq b_{l} \leq p^{k}-1$ is the unique integer so that $b_{l} \equiv l q^{k} \bmod p^{k}$, and $\psi_{k}(\theta)=\prod_{\gamma=0}^{k-1} \psi\left(p^{\gamma} q^{k-1-\gamma} \theta\right)$. Then

$$
\sup _{\|f\|_{2}^{2}=1} \sum_{t=0}^{q^{k}-1}\left\|R_{p^{k}} M_{\Psi_{k}} \mathcal{L}_{\zeta_{k}^{t}} f\right\|_{2}^{2} \approx\left\|U_{k}\right\|, \quad \zeta_{k}=e^{i 2 \pi / p^{k}}
$$

where $U_{k}$ is a positive definite $q^{k}$ by $q^{k}$ matrix on $\mathbf{C}^{q^{k}}$ whose ij-entry is

$$
\frac{1}{p^{k}} \sum_{l=0}^{p^{k}-1} \psi_{k}\left(\frac{2 \pi l}{p^{k}}+\frac{2 \pi i}{q^{k}}\right) \psi_{k}\left(\frac{2 \pi l}{p^{k}}+\frac{2 \pi j}{q^{k}}\right), \quad 0 \leq i, j \leq q^{k}-1 .
$$

Proof. By applying the same technique used in the proof of Theorem 2.3 we see that

$$
\sup _{\|f\|_{2}^{2}=1} \sum_{t=0}^{q^{k}-1}\left\|R_{p^{k}} M_{\Psi_{k}} \mathcal{L}_{\zeta_{k}^{t}} f\right\|_{2}^{2}=\left\|\mathcal{W}_{k}\right\|^{2}
$$

where $\mathcal{W}_{k}$ is the operator on $\bigoplus_{1}^{q^{k}} L^{2}(\mathbf{T})$ defined by

$$
\mathcal{W}_{k}\left(f_{0}, \cdots, f_{q^{k}-1}\right):=\left(g_{0}, \cdots, g_{q^{k}-1}\right), \quad g_{t}=R_{p^{k}} M_{\Psi_{k}} \mathcal{L}_{\zeta_{k}^{t}} f_{0},
$$

with adjoint

$$
\mathcal{W}_{k}^{*}\left(f_{0}, \cdots, f_{q^{k}-1}\right):=(g, 0,0, \cdots, 0), \quad g=\sum_{t=0}^{q^{k}-1} \mathcal{L}_{\bar{\zeta}_{k}^{t}} M_{\Psi_{k}} C_{p^{k}} f_{t},
$$

for $\left(f_{0}, \cdots, f_{q^{k}-1}\right) \in \bigoplus_{1}^{q^{k}} L^{2}(\mathbf{T})$. Since $\left\|\mathcal{W}_{k}\right\|^{2}=\left\|\mathcal{W}_{k} \mathcal{W}_{k}^{*}\right\|$, it follows from (2.3) that $\left\|\mathcal{W}_{k}\right\|^{2}=\left\|\mathcal{Q}_{k}\right\|$, where

$$
\mathcal{Q}_{k}\left(f_{0}, \cdots, f_{q^{k}-1}\right):=\left(g_{0}, \cdots, g_{q^{k}-1}\right), \quad g_{t}=\sum_{s=0}^{q^{k}-1}\left(R_{p^{k}}\left(\Psi_{k, t} \Psi_{k, s}\right)\right) f_{s},
$$

and $\Psi_{k, t}(\theta)=\Psi_{k}\left(\theta-\frac{2 \pi t}{p^{k}}\right), \theta \in \mathbf{R}$.

Now suppose that $0 \leq x \leq p^{k}-1$ and $0 \leq y \leq p^{k}-1$ are integers so that $x \equiv y q^{k}$ $\bmod p^{k}$, then by the Chinese remainder theorem, the number

$$
y=\frac{p^{k} z+x}{q^{k}}
$$


satisfies $x \equiv y q^{k} \bmod p^{k}$, where $z$ satisfies $-x \equiv z p^{k} \bmod q^{k}$. On the other hand, since $\left(p^{k}, q^{k}\right)=1$, there exist $\alpha_{k}, \beta_{k} \in \mathbf{Z}$ such that $\alpha_{k} p^{k}+\beta_{k} q^{k}=1$. Therefore, if we choose $z=-\alpha_{k} x$, the number

$$
y=\frac{\left(1-\alpha_{k} p^{k}\right) x}{q^{k}}=\beta_{k} x
$$

satisfies $x \equiv y q^{k} \bmod p^{k}$, and this means that $\Psi_{k}\left(\frac{\theta}{p^{k}}+\frac{2 \pi x}{p^{k}}\right)=\psi_{k}\left(\frac{2 \pi \beta_{k} x}{p^{k}}\right)$ for $\theta \in[0,2 \pi)$. So for $0 \leq l \leq p^{k}-1$ and $0 \leq t \leq q^{k}-1$, we have

$$
\begin{aligned}
\Psi_{k, t}\left(\frac{\theta+2 \pi l}{p^{k}}\right) & =\Psi_{k}\left(\frac{\theta+2 \pi l}{p^{k}}-\frac{2 \pi t}{p^{k}}\right)=\psi_{k}\left(\frac{2 \pi \beta_{k} l}{p^{k}}-\frac{2 \pi \beta_{k} t}{p^{k}}\right) \\
& =\psi_{k}\left(\frac{2 \pi \beta_{k} l}{p^{k}}+\frac{2 \pi \alpha_{k} t}{q^{k}}-\frac{2 \pi t}{p^{k} q^{k}}\right) \\
& \approx \psi_{k}\left(\frac{2 \pi \beta_{k} l}{p^{k}}+\frac{2 \pi \alpha_{k} t}{q^{k}}\right) \quad(0 \leq \theta<2 \pi)
\end{aligned}
$$

uniformly in $k$ by Lemma 3.1 since $0 \leq \frac{2 \pi t}{p^{k} q^{k}} \leq \frac{2 \pi}{p^{k}}$ if $0 \leq t \leq q^{k}-1$. Hence

$$
R_{p^{k}}\left(\Psi_{k, t} \Psi_{k, s}\right) \approx \frac{1}{p^{k}} \sum_{l=0}^{p^{k}-1} \psi_{k}\left(\frac{2 \pi l}{p^{k}}+\frac{2 \pi \alpha_{k} t}{q^{k}}\right) \psi_{k}\left(\frac{2 \pi l}{p^{k}}+\frac{2 \pi \alpha_{k} s}{q^{k}}\right)
$$

uniformly in $k$ since the fact that $\left(\beta_{k}, p^{k}\right)=1$ implies that $\left\{\beta_{k} l: 0 \leq l \leq p^{k}-1\right\}$ is simply a rearrangement of $\left\{0,1,2, \cdots, p^{k}-1\right\}$. By the same reason, since $\left(\alpha_{k}, q^{k}\right)=$ $1,\left\{\alpha_{k} t: 0 \leq t \leq q^{k}-1\right\}=\left\{0,1,2, \cdots, q^{k}-1\right\}$, and this completes the proof.

An $n \times n$ matrix $A=\left(a_{i j}\right), 1 \leq i, j \leq n$, is called a Toeplitz matrix if $a_{i j}=a_{j-i}$ for all $i, j$. Toeplitz matrices have found a wide variety of applications in areas including analytic functions, stochastic process and statistics (see, for example, [5]). The next result is interesting since it states that the spectral radius of $S_{\varphi}(m, n)$ can be expressed in terms of the limit of roots of the norms of some finite Toeplitz matrices.

Theorem 3.3. Let $\varphi$ be continuous on $\mathbf{T}$ and $m>n$. Let $m=p r, n=q r$, $(p, q)=1, \psi=R_{r} \varphi$ and $\psi_{k}(\theta)=\prod_{\gamma=0}^{k-1} \psi\left(p^{\gamma} q^{k-1-\gamma} \theta\right)$. Then

$$
r\left(S_{\varphi}(m, n)\right)=\lim _{k \rightarrow \infty}\left\|S_{k}\right\|^{\frac{1}{2 k}}
$$

if $\psi>0$, where $S_{k}$ is a real symmetric $q^{k} \times q^{k}$ matrix whose ij-entry is

$$
\frac{1}{p^{k} q^{k}} \sum_{l=0}^{p^{k}-1} \psi_{k}\left(\frac{2 \pi l}{p^{k}}+\frac{2 \pi i}{q^{k}}\right) \psi_{k}\left(\frac{2 \pi l}{p^{k}}+\frac{2 \pi j}{q^{k}}\right), \quad 0 \leq i, j \leq q^{k}-1 .
$$

Furthermore, the norm of $S_{k}$ is comparable to that of a real symmetric Toeplitz matrix $T_{k}$ on $\mathbf{C}^{q^{k}}$ for each $k$. Specifically, the ij-entry for $T_{k}$ is

$$
\frac{1}{p^{k} q^{k}} \sum_{l=0}^{p^{k}-1} \psi_{k}\left(\frac{2 \pi l}{p^{k}}\right) \psi_{k}\left(\frac{2 \pi\left(l+\beta_{j-i}\right)}{p^{k}}\right), \quad 0 \leq i, j \leq q^{k}-1
$$

where $\beta_{s}=\left[\frac{p^{k} s}{q^{k}}\right](s \in \mathbf{Z})$, and $[x]$ is the smallest integer less than or equal to $x$. 
Proof. Since $S_{\varphi}(m, n)^{k}=S_{\psi_{k}}\left(p^{k}, q^{k}\right)$, we have

$$
\begin{aligned}
& \left\|S_{\varphi}(m, n)^{k} f\right\|_{2}^{2}=\frac{1}{p^{2 k}} \int_{0}^{2 \pi}\left|\sum_{l=0}^{p^{k}-1} \psi_{k}\left(\frac{\theta+2 \pi l}{p^{k}}\right) f\left(\frac{q^{k}(\theta+2 \pi l)}{p^{k}}\right)\right|^{2} \frac{d \theta}{2 \pi} \\
& =\frac{1}{p^{2 k} q^{k}} \sum_{t=0}^{q^{k}-1} \int_{0}^{2 \pi}\left|\sum_{l=0}^{p^{k}-1} \psi_{k}\left(\frac{\theta+2 \pi t}{p^{k} q^{k}}+\frac{2 \pi l}{p^{k}}\right) f\left(\frac{\theta+2 \pi\left(t+l q^{k}\right)}{p^{k}}\right)\right|^{2} \frac{d \theta}{2 \pi} \\
& \approx \frac{1}{p^{2 k} q^{k}} \sum_{t=0}^{q^{k}-1} \int_{0}^{2 \pi}\left|\sum_{l=0}^{p^{k}-1} \psi_{k}\left(\frac{2 \pi l}{p^{k}}\right) f\left(\frac{\theta+2 \pi\left(t+l q^{k}\right)}{p^{k}}\right)\right|^{2} \frac{d \theta}{2 \pi} \\
& =\frac{1}{q^{k}} \sum_{t=0}^{q^{k}-1}\left\|R_{p^{k}} M_{\Psi_{k}} C_{\zeta_{k}^{t}} f\right\|_{2}^{2},
\end{aligned}
$$

for any $f \geq 0$ in $L^{2}(\mathbf{T})$, where $\Psi_{k}$ and $\zeta_{k}$ are defined in Lemma 3.2 Note also that the last equation follows from the fact that $\left(p^{k}, q^{k}\right)=1$. Therefore, by Lemma 3.2 $\left\|S_{\varphi}(m, n)^{k}\right\|^{2} \approx\left\|S_{k}\right\|$ since $q^{k} S_{k}=U_{k}$ (it is enough to assume that $f \geq 0$ since $\psi \geq 0)$.

Now let $0 \leq i \leq q^{k}-1$. Suppose that $\frac{2 \pi \alpha}{p^{k}} \leq \frac{2 \pi i}{q^{k}}<\frac{2 \pi(\alpha+1)}{p^{k}}$ for some integer $0 \leq \alpha \leq p^{k}-1$; then $\left[\frac{p^{k} i}{q^{k}}\right]-1<\alpha \leq\left[\frac{p^{k} i}{q^{k}}\right]$. It follows that

$$
\psi_{k}\left(\frac{2 \pi l}{p^{k}}+\frac{2 \pi i}{q^{k}}\right) \approx \psi_{k}\left(\frac{2 \pi l}{p^{k}}+\frac{2 \pi \beta_{i}}{p^{k}}\right),
$$

uniformly in $k$, where $\beta_{i}=\left[\frac{p^{k} i}{q^{k}}\right]$, and therefore, uniformly in $k$, we have

$$
\begin{aligned}
\sum_{l=0}^{p^{k}-1} \psi_{k}\left(\frac{2 \pi l}{p^{k}}+\frac{2 \pi i}{q^{k}}\right) \psi_{k}\left(\frac{2 \pi l}{p^{k}}+\frac{2 \pi j}{q^{k}}\right) & \approx \sum_{l=0}^{p^{k}-1} \psi_{k}\left(\frac{2 \pi l}{p^{k}}+\frac{2 \pi \beta_{i}}{p^{k}}\right) \psi_{k}\left(\frac{2 \pi l}{p^{k}}+\frac{2 \pi \beta_{j}}{p^{k}}\right) \\
& =\sum_{l=0}^{p^{k}-1} \psi_{k}\left(\frac{2 \pi l}{p^{k}}\right) \psi_{k}\left(\frac{2 \pi l}{p^{k}}+\frac{2 \pi\left(\beta_{j}-\beta_{i}\right)}{p^{k}}\right) \\
& \approx \sum_{l=0}^{p^{k}-1} \psi_{k}\left(\frac{2 \pi l}{p^{k}}\right) \psi_{k}\left(\frac{2 \pi l}{p^{k}}+\frac{2 \pi\left(\beta_{j-i}\right)}{p^{k}}\right) \\
& \approx \sum_{l=0}^{p^{k}-1} \psi_{k}\left(\frac{2 \pi l}{p^{k}}\right) \psi_{k}\left(\frac{2 \pi l}{p^{k}}+\frac{2 \pi(j-i)}{q^{k}}\right)
\end{aligned}
$$

Notice that the equations above follow from the definition of $\beta_{i}$, the property of the Gauss function $[x]$ and the fact that $\psi_{k}$ is $2 \pi$ periodic. So far we have shown that if $\psi>0$, then $\left\|S_{\varphi}(m, n)^{k}\right\| \approx\left\|S_{k}\right\|^{\frac{1}{2}} \approx\left\|T_{k}\right\|^{\frac{1}{2}}$ and $T_{k}$ is a Toeplitz matrix on $\mathbf{C}^{q^{k}}$ for all $k$. Hence,

$$
r\left(S_{\varphi}(m, n)\right)=\lim _{k \rightarrow \infty}\left\|S_{\varphi}(m, n)^{k}\right\|^{\frac{1}{k}}=\lim _{k \rightarrow \infty}\left\|S_{k}\right\|^{\frac{1}{2 k}}=\lim _{k \rightarrow \infty}\left\|T_{k}\right\|^{\frac{1}{2 k}} .
$$

Let $\varphi \in L^{\infty}(\mathbf{T})$ and let $P$ be the projection from $L^{2}(\mathbf{T})$ onto $H^{2}$, where $H^{2}$ is the so-called Hardy space on the unit disc, identified as the closed span of $\left\{e^{i k \theta}\right.$ : $k \geq 0\}$ in $L^{2}(\mathbf{T})$. The operator $T_{\varphi}: H^{2} \rightarrow H^{2}$ defined by $T_{\varphi}=P M_{\varphi}$ is called the 
Toeplitz operator with symbol $\varphi$. Now let $\varphi \sim \sum a_{k} e^{i k \theta}$. It is well-known that the representing matrix of $T_{\varphi}$ with respect to the standard basis $\left\{e^{i k \theta}: k \geq 0\right\}$ on $H^{2}$ is $\left\{a_{j-i}\right\}, i, j \geq 0$ (which is sometimes called an "infinite" Toeplitz matrix) and we have $\left\|T_{\varphi}\right\|=\|\varphi\|_{\infty}$. Since it is easy to see that any finite Toeplitz matrix $\left\{a_{j-i}\right\}$, $0 \leq i, j \leq n-1$ is an $n \times n$ minor of the infinite Toeplitz matrix $\left\{a_{j-i}\right\}, i, j \geq 0$ and since all entries of $T_{k}$ are positive, we have

$$
\left\|T_{k}\right\| \leq\left\|T_{\varphi}\right\|=\frac{2}{p^{k} q^{k}} \sum_{i=0}^{q^{k}-1} \sum_{l=0}^{p^{k}-1} \psi_{k}\left(\frac{2 \pi l}{p^{k}}\right) \psi_{k}\left(\frac{2 \pi\left(l+\beta_{i}\right)}{p^{k}}\right)-\frac{1}{p^{k} q^{k}} \sum_{l=0}^{p^{k}-1} \psi_{k}\left(\frac{2 \pi l}{p^{k}}\right)^{2}
$$

for all $k$, where the symbol $\varphi \sim \sum a_{j} e^{i j \theta}$ for the Toeplitz operator $T_{\varphi}$ in the above inequality is given by $a_{j}=\frac{1}{p^{k} q^{k}} \sum_{l=0}^{p^{k}-1} \psi_{k}\left(\frac{2 \pi l}{p^{k}}\right) \psi_{k}\left(\frac{2 \pi\left(l+\beta_{j}\right)}{p^{k}}\right)$ for $-q^{k}+1 \leq j \leq q^{k}-1$ and $a_{j}=0$ if else. Here the author would like to refer the readers to [3], which is an excellent source of reference on the subjects concerning the Toeplitz operator. On the other hand, since $T_{k}$ is positive definite, $\left\|T_{k}\right\|$ equals $\sup \left\{\left\langle T_{k} x, x\right\rangle: x \in\right.$ $\left.\mathbf{C}^{q^{k}},\|x\|_{2}=1\right\}$. So in particular, if $x=\left(q^{-\frac{k}{2}}, \cdots, q^{\frac{-k}{2}}\right)^{*}$, then $\|x\|_{2}=1$ and $\left\|T_{k}\right\| \geq\left\langle T_{k} x, x\right\rangle$, i.e.,

$$
\left\|T_{k}\right\| \geq \frac{1}{p^{k} q^{2 k}} \sum_{i, j=0}^{q^{k}-1} \sum_{l=0}^{p^{k}-1} \psi_{k}\left(\frac{2 \pi\left(l+\beta_{i}\right)}{p^{k}}\right) \psi_{k}\left(\frac{2 \pi\left(l+\beta_{j}\right)}{p^{k}}\right) .
$$

Now fix an $i$. For any $0 \leq j \leq q^{k}-1$, we have

$$
\begin{aligned}
\sum_{l=0}^{p^{k}-1} \psi_{k}\left(\frac{2 \pi\left(l+\beta_{i}\right)}{p^{k}}\right) \psi_{k}\left(\frac{2 \pi\left(l+\beta_{j}\right)}{p^{k}}\right) & =\sum_{l=0}^{p^{k}-1} \psi_{k}\left(\frac{2 \pi\left(l+\beta_{i-j}\right)}{p^{k}}\right) \psi_{k}\left(\frac{2 \pi l}{p^{k}}\right) \\
& =\sum_{l=0}^{p^{k}-1} \psi_{k}\left(\frac{2 \pi l}{p^{k}}\right) \psi_{k}\left(\frac{2 \pi\left(l+\beta_{q^{k}-(i-j)}\right)}{p^{k}}\right)
\end{aligned}
$$

since $\psi_{k}$ is $2 \pi$ periodic. Therefore $t_{k} \leq\left\|T_{k}\right\| \leq 2 t_{k}$, where

$$
t_{k}=\frac{1}{p^{k}} \sum_{l=0}^{p^{k}-1}\left(\frac{1}{q^{k}} \sum_{i=0}^{q^{k}-1} \psi_{k}\left(\frac{2 \pi\left(l+\beta_{i}\right)}{p^{k}}\right)\right)^{2}
$$

These observations lead to one of the main results of this paper:

Theorem 3.4. Let $\varphi$ be a continuous function on $\mathbf{T}$. If $m>n$, then

$$
r\left(S_{\varphi}(m, n)\right)=\lim _{k \rightarrow \infty}\left\|R_{p^{k}}\left(R_{q^{k}} \psi_{k}\right)^{2}\right\|_{\infty}^{\frac{1}{2 k}}
$$

if $\psi=\psi_{1}>0$, where $p, q$ and $\psi_{k}$ are defined as before.

Proof. As we have seen preceding the theorem, it suffices to show that $t_{k} \approx$ $\left\|R_{p^{k}}\left(R_{q^{k}} \psi_{k}\right)^{2}\right\|_{\infty}$ by Theorem 3.3. Now since $C_{q^{k}}$ is also an isometry on $C(\mathbf{T})$, we have, with the help of Lemma 2.1 that $\left\|R_{p^{k}}\left(R_{q^{k}} \psi_{k}\right)^{2}\right\|_{\infty}=\left\|R_{p^{k}}\left(C_{q^{k}} R_{q^{k}} \psi_{k}\right)^{2}\right\|_{\infty}$. On the other hand, since $R_{p^{k}}\left(C_{q^{k}} R_{q^{k}} \psi_{k}\right)^{2} \in C(\mathbf{T})$ and $\mathbf{T}$ is compact, there exists $\theta_{k} \in[0,2 \pi)$ such that $\left\|R_{p^{k}}\left(C_{q^{k}} R_{q^{k}} \psi_{k}\right)^{2}\right\|_{\infty}=\left(R_{p^{k}}\left(C_{q^{k}} R_{q^{k}} \psi_{k}\right)^{2}\right)\left(\theta_{k}\right)$, i.e. for each $k$, we have

$$
\left\|R_{p^{k}}\left(R_{q^{k}} \psi_{k}\right)^{2}\right\|_{\infty}=\frac{1}{p^{k}} \sum_{l=0}^{p^{k}-1}\left(\frac{1}{q^{k}} \sum_{i=0}^{q^{k}-1} \psi_{k}\left(\frac{\theta_{k}+2 \pi l}{p^{k}}+\frac{i}{q^{k}}\right)\right)^{2}
$$


by (2.2). Hence, $t_{k} \approx\left\|R_{p^{k}}\left(R_{q^{k}} \psi_{k}\right)^{2}\right\|_{\infty}$ follows from the fact

$$
\psi_{k}\left(\frac{2 \pi\left(l+\beta_{i}\right)}{p^{k}}\right) \approx \psi_{k}\left(\frac{2 \pi l}{p^{k}}+\frac{i}{q^{k}}\right) \approx \psi_{k}\left(\frac{\theta_{k}+2 \pi l}{p^{k}}+\frac{i}{q^{k}}\right)
$$

uniformly in $k$ by Lemma 3.1

Remark. When $q=1, S_{\varphi}(m, n)$ reduces to $S_{\psi}(p, 1)=R_{p} M_{\psi}$. In this special case, the spectral radius formula of $S_{\varphi}(m, n)$ in the form given in Theorem 3.4 can be derived directly from repeated applications of (2.4) and Lemma 2.1. The author believes that the formula presented in Theorem 3.3 is true for all nonnegative $\psi$, but has not been able prove it at this stage. On the other hand, the author has also come to believe that the possibility of the strict inequality $r\left(S_{\varphi}(m, n)\right)<$ $r\left(S_{|\varphi|}(m, n)\right)$ exists (i.e., Theorem 3.4 may be invalid) if $\psi$ is no longer nonnegative. The reason being that since if $q>1$, then $C_{q^{k}}$ does not map $L^{\infty}(\mathbf{T})$ onto itself and, consequently, there may not exist $f \in L^{2}(\mathbf{T})$ such that

$$
R_{p^{k}} M_{\psi_{k}} C_{q^{k}} f=R_{p^{k}}\left(\psi_{k} C_{q^{k}} f\right)=R_{p^{k}}\left(\left|\psi_{k}\right| C_{q^{k}}|f|\right)=R_{p^{k}} M_{\left|\psi_{k}\right|} C_{q^{k}}|f| .
$$

\section{REFERENCES}

[1] A. Cohen and I. Daubechies, A stability criterion for biorthogonal wavelet bases and their related subband coding scheme, Duke Math. J., 68, 1992, pp. 313-335. MR 94b:94005

[2] A. Cohen and I. Daubechies, A new technique to estimate the regularity of refinable functions, Revista Mathematica Iberoamericana, 12, 1996, pp. 527-591. MR 97g:42025

[3] R.G. Douglas, Banach algebra techniques in operator theory, Academic Press, New York, 1972. MR 50:14335

[4] T. Goodman, C. Micchelli and J. Ward, Spectral radius formula for subdivision operators, Recent Advances in Wavelet Analysis, ed. L. Schumaker and G. Webb, Academic Press, 1994, pp. 335-360. MR 94m:47076

[5] U. Grenander and G. Szegö, Toeplitz forms and their applications, Chelsea Publishing Co., New York, 1955.

[6] R. A. Horn and C. R. Johnson, Matrix analysis, Cambridge University Press, 1985. MR 87e:15001

[7] G. Strang, Eigenvalues of $(\downarrow 2) H$ and convergence of the cascade algorithm, IEEE Trans. Sig. Proc., 44, 1996.

[8] W. Sweldens and P. Schröder, Building Your Own Wavelets at Home, Wavelets in Computer Graphics, ACMSIGGRAPH Course Notes, 1996

[9] L. Villemoes, Wavelet analysis of refinement equations, SIAM J. Math. Analysis, 25, no. 5, 1994, pp. 1433-1460. MR 96f:39009

[10] P. Zizler, Norm of sampling operators, Linear Algebra and its Application, 277, 1998, pp. 291-298. MR 99c:47030

Department of Applied Mathematics, National Sun Yat-Sen University, Kaohsiung, TAIWAN

E-mail address: hom@math.nsysu.edu.tw 